\title{
A dilated MPC control strategy for LPV linear systems
}

\author{
Alessandro Casavola, Domenico Famularo, Giuseppe Franzè and Emanuele Garone
}

\begin{abstract}
A novel model predictive control (MPC) strategy for input-saturated polytopic linear parameter varying (LPV) discrete-time systems is proposed. It is assumed that the scheduling vector of the plant parametrization is available at each time instant. A new dilation technique and parameter varying Lyapunov functions are exploited to reduce conservativeness of existing robust MPC paradigms. The contribution of this paper is to extend the RHC scheme presented in [5] to the general case of control horizons of arbitrary length $N$. Feasibility and closed-loop stability of this strategy are proved and two final numerical examples presented in order to show performance improvements w.r.t. preexisting techniques.
\end{abstract}

\section{INTRODUCTION}

Over the last decade Model Predictive Control (MPC) have attracted significant attention mainly due to its ability to handle constraints on inputs and outputs. Actually, one of most critical open problems (see [14], [10], [18], [15] and references therein) is how to achieve non-conservative regulation strategies at the expense of modest computational burdens.

Recently, starting from an idea of Bernussou et al. [8], [7], dilation techniques have been used to derive more efficient MPC control laws by introducing more flexible parameter dependent Lyapunov functions (see [6] and [13]) instead of traditional quadratic stability approaches. Such a paradigm has been extended to the case of Linear Parameter Varying (LPV) systems expressed via multi-model or affine representation in [5], by exploiting the property that the scheduling vector is available in real time.

The contribution of this paper is to develop a MPC control strategy for input saturated multi-model LPV systems based on dilation techniques. It will be shown that improved control performance and a larger feasible set of initial states are achieved w.r.t. traditional LPV receding-horizon control algorithms (see [12], [4] and references therein).

We consider the general case of arbitrary $N$-steps control horizons and assume the LPV scheduling vector measurable at each time instant. A bank of state-feedback gains, computed off-line, is used as primal control law over which a sequence of $N$ free control offsets, representing the degrees of freedom of the underlying optimization problem, is superimposed.

This work has been supported by MIUR Project Fault Detection and Diagnosis, Control Reconfiguration and Performance Monitoring in Industrial Process

Alessandro Casavola, Giuseppe Franzè and Emanuele Garone are with the Dipartimento di Elettronica, Informatica e Sistemistica, Universitá degl Studi della Calabria, Via Pietro Bucci, Cubo 42-C, Rende (CS), 87036, ITALY \{casavola, franze, egarone\}@deis.unical.it

Domenico Famularo is with DIMET, Facoltá di Ingegneria, Universitá degli Studi di Reggio Calabria, Reggio Calabria, Via Graziella, 89100, ITALY famularo@ing.unirc.it
The feedback gains are computed off-line by means of the procedure described in [5], whereas the control moves are computed on-line by solving a semi-definite constrained programming problem involving linear matrix inequalities. The closed-loop stability and feasibility properties of the solution can be proved via standard arguments and are here summarized. Two numerical examples are considered and comparisons with two MPC strategies, a LPV algorithm which does not make use of the dilation techniques and an extension of a robust dilated MPC scheme proposed by [6], are shown.

The paper is organized as follows: the problem is stated in Section II where previous results are also described. In Section III, the novel dilated LPV algorithm is presented, all required LMI conditions are derived and the feasibility and stability properties proved. Two numerical experiments are reported in Section IV and some conclusions end the paper.

\section{NOTATION}

Given a matrix $X \in \mathbb{R}^{n \times n}$, we will denote with $X_{i i}, i=$ $1, \ldots, n$, the $i$-th diagonal entry.

Given a symmetric matrix $P=P^{T} \in \mathbb{R}^{n \times n}$, the inequality $P>0(P \geq 0)$ denotes matrix positive definiteness (semi-definiteness). Given two symmetric matrices $P, Q$, the inequality $P>Q(P \geq Q)$ indicates that $P-Q>0$, $(P-Q \geq 0)$.

Given a vector $x \in \mathbb{R}^{n}$, the standard 2-norm is denoted by $\|x\|_{2}^{2}=x^{T} x$ whereas $\|x\|_{P}^{2} \triangleq x^{T} P x$ denotes the vector $P$-weighted 2-norm.

The notation $\hat{v}_{k}(t) \triangleq v(t+k \mid t), \quad k \geq 0$ will be used to define the $k$-step ahead prediction of a generic system variable $v$ from $t$ onward under specified initial state and input scenario.

\section{Problem statement}

Let us consider the following discrete-time LPV system

$$
\begin{cases}x(t+1) & =A(p(t)) x(t)+B(p(t)) u(t) \\ y(t) & =C x(t)\end{cases}
$$

with $x(t) \in \mathbf{R}^{n}$ denoting the state, $u(t) \in \mathbf{R}^{m}$ the plant input and $y(t) \in \mathbf{R}^{p}$ the output. The plant matrices have the following structure:

$$
A(p)=\sum_{j=1}^{l} p_{j} A_{j}, \quad B(p)=\sum_{j=1}^{l} p_{j} B_{j},
$$

with $p=\left[p_{1}, p_{2}, \ldots, p_{l}\right]^{T} \in \mathcal{P}$ in the unit simplex

$$
\mathcal{P}:=\left\{\sum_{j=1}^{l} p_{j}=1,0 \leq p_{j} \leq 1\right\} .
$$


We assume hereafter

- LPV - $p(t)$ is measurable at each time instant.

We will denote as $\mathcal{P}^{r}$ and $\mathcal{P}^{*}$ the sets of all possible $\mathcal{P}$ valued sequences of $r+1$ and arbitrary length, respectively,

$$
\mathcal{P}^{r}:=\left\{\{p(t)\}_{t=0}^{r}: p(t) \in \mathcal{P}\right\}, \quad \mathcal{P}^{*}:=\lim _{r \rightarrow \infty} \mathcal{P}^{r} .
$$

Moreover, let the plant input be subject to componentwise saturation constraints

$$
\left|u_{j}(t)\right| \leq u_{j, \max } t \geq 0, j=1, \ldots, m,
$$

The aim is to find a state-feedback regulation strategy $u(t)=$ $g(x(t))$ which asymptotically stabilizes (1)-(3) subject to (5).

Consider now, for a generic command sequence $u(\cdot)$, the following quadratic performance index

$$
J(x, u(\cdot)):=\max _{p(\cdot) \in \mathcal{P}^{*}} \sum_{t=0}^{\infty}\left\{\|x(t)\|_{R_{x}}^{2}+\|u(t)\|_{R_{u}}^{2}\right\} .
$$

with $R_{u}=R_{u}^{T}>0, R_{x}=R_{x}^{T}>0$ suitable input and state weights.

If the control strategy has a linear state-feedback form $u=F x$, an upper bound to the cost (6) is given by (see [10] for details)

$$
J(x(0), F x(\cdot)) \leq x(0)^{T} P x(0)
$$

with $P=P^{T}>0$ satisfying the (robust) quadratic stabilization conditions [9] of the polytopic model (1)-(3)

$$
A_{F, j}^{T} P A_{F, j}-P+F^{T} R_{u} F+R_{x} \leq 0, \quad j=1, \ldots, l,
$$

where $A_{F, j}:=A_{j}+B_{j} F, R_{x}=C^{T} R_{y} C$.

Recently, for the sake of reducing conservativeness, conditions (7)-(8) have been approached (see [5]) in a receding horizon fashion by defining a linearly parameter dependent Lyapunov function (PDLF) [8]

$$
V(x(t))=x^{T}(t) P(p(t)) x(t)
$$

with

$$
P(p(t))=\sum_{j=1}^{l} p_{j}(t) P_{j},
$$

and the state feedback control law

$$
\hat{u}(t+k \mid t)=F(p(t)) \hat{x}(t+k \mid t), \quad k \geq 0
$$

with

$$
F(p(t))=\sum_{j=1}^{l} p_{j}(t) F_{j}, \quad j=1, \ldots, l
$$

Remark 1 - Note that, given the $p$-parametric expression of $P$ in (10), the upper bound (7) becomes

$$
J(x(0), F(p(t)) x(\cdot)) \leq \max _{j=1, \ldots, l} x(0)^{T} P_{j} x(0)
$$

with $P_{j}=P_{j}^{T}>0, j=1, \ldots, l$

Moreover, the vertices $P_{j}, j=1, \ldots, l$ are shaping matrices for the following family of ellipsoidal sets

$$
\begin{aligned}
\mathcal{E}\left(P_{j}, \gamma\right):= & \left\{x \in \mathbf{R}^{n} \mid x^{T} P_{j} x \leq \gamma, \gamma \geq 0\right\} \\
& j=1, \ldots, l
\end{aligned}
$$

whose intersection

$$
\bigcap_{j=1}^{l} \mathcal{E}\left(P_{j}, \gamma\right)
$$

can be proved to be a robust positively invariant region for the polytopic system (1)-(3) under the state-feedback $F$.

In [5], the problem of determining at each time step $t$ a stabilizing state-feedback $F(p(t))$, such that input constraints (5) are satisfied, has been solved by resorting to the following algorithm:

$$
\min _{Y_{j}, G, Q_{j}} \gamma
$$

subject to

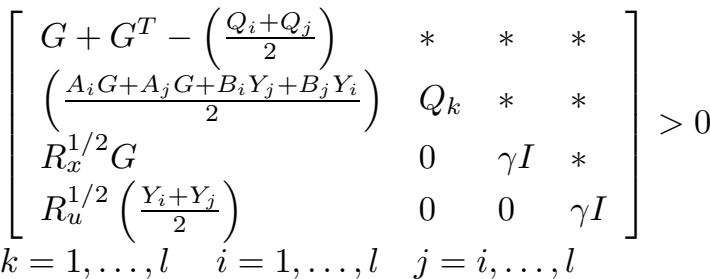

$$
\begin{aligned}
& {\left[\begin{array}{ll}
1 & x^{T}(t) \\
x(t) & Q_{j}
\end{array}\right]>0} \\
& {\left[\begin{array}{ll}
X & Y_{j} \\
Y_{j}^{T} & G^{T}+G-Q_{j}
\end{array}\right] \geq 0} \\
& j=1, \ldots, l \\
& X_{k k} \leq u_{k, \max }^{2} \quad k=1, \ldots, m,
\end{aligned}
$$

with $P_{j}=\gamma Q_{j}^{-1}, F_{j}=Y_{j} G^{-1}$, the matrix $G \in \mathbf{R}^{n \times n}$ acting like an additional degree of freedom.

Note that under (11), the closed-loop polytopic system family

$$
A_{F}(t):=A(p(t))+B(p(t)) F(p(t))
$$

belongs to the convex hull

$$
A_{F}(t) \in \operatorname{co}\left\{\bar{A}_{i j}, \quad i=1, \ldots, l, \quad j=i, i+1, \ldots, l\right\}
$$

whose vertices are

$$
\begin{aligned}
& \bar{A}_{i j}:=\frac{\left(A_{i}+B_{i} F_{j}\right)+\left(A_{j}+B_{j} F_{i}\right)}{2}, \\
& i=1, \ldots, l, \quad j=i, i+1, \ldots, l
\end{aligned}
$$

Here we will consider the following generalization of the control law (11)

$\hat{u}(\cdot \mid t)= \begin{cases}F(p(t)) \hat{x}(t+k \mid t)+\hat{c}(t+k \mid t), & k=0,1, \ldots, N-1 \\ F(p(t)) \hat{x}(t+k \mid t), & k \geq N\end{cases}$

where the term $\hat{c}(t+k \mid t)$ represents an additional degree of freedom whose role is that of ameliorating the control performances over (11). 


\section{DilATED MPC SCHEME}

In this section we will determine the conditions which guarantee a convenient upper-bound to the quadratic cost (6) and ensure satisfaction of the prescribed constraints under the control law (21).

First of all, observe that the convex hulls structure of $k$ step ahead state predictions starting from $x:=x(t)$ are given by

$$
\begin{gathered}
\mathfrak{X}^{t \mid t} \triangleq\{x\} \\
\mathfrak{X}^{t+1 \mid t} \triangleq\left\{A_{F}(t) x+B(p(t)) \hat{c}(t \mid t), \forall p \in \mathcal{P}^{0}(t)\right\}^{1} \\
\mathfrak{X}^{t+k \mid t \triangleq} \operatorname{co}\left\{A_{F}(t+k-1) z+B(p(t+k-1)) \hat{c}(t+k-1 \mid t),\right. \\
\left.\forall p \in \mathcal{P}^{k-1}(t), \forall z \in \operatorname{vert}\left\{\mathfrak{X}^{t+k-1 \mid t}\right\}\right\}
\end{gathered}
$$

Each vertex of $\mathfrak{X}^{t+k \mid t}$, for fixed $F$, is an affine function of the free term $c(t+k-1 \mid t)$. This is the key point to derive a semidefinite programming algorithm which solves the problem. According to the strategy (21), a convenient quadratic performance index is

$$
\begin{aligned}
& V\left(x(t), P_{j}, F_{j}, \hat{c}(\cdot \mid t)\right):= \\
& \sum_{k=0}^{N-1}\left\{\max _{z(k) \in \operatorname{vert}\left\{\mathfrak{X}^{t+k \mid t}(x(t))\right\}}\|z(k)\|_{R_{x}}^{2}+\|c(t+k \mid t)\|_{R_{u}}^{2}\right\} \\
& +\max _{z \in \operatorname{vert}\left\{\mathfrak{X}^{t+N \mid t}(x(t))\right\}, \quad P_{j}, j=1, \ldots, l}\|z\|_{P_{j}}^{2}
\end{aligned}
$$

with $P_{j}=\gamma Q_{j}^{-1}, j=1, \ldots, l$, solution of the LMIs (17)-(19) with $x=x(t)$. Then, the overall algorithm is as follows:

\section{Algorithm - DL-LPV}

- Initialization:

Given $x(0)$ find

$$
\left[\left\{Q_{j}, Y_{j}\right\}_{j=1}^{l}, G, \gamma\right]:=\arg \min _{Y_{j}, Q_{j}>0, \gamma>0} \gamma
$$

subject to LMIs (17)-(19).

$P_{j}:=\gamma Q_{j}^{-1}, F_{j}:=Y_{j} G^{-1}, j=1, \ldots, l, F(0):=$ $\sum_{i=1}^{l} p_{i}(0) F_{j}$

- Generic Step:

1. For any $t \geq 0$, given $x(t),\left\{P_{j}, F_{j}\right\}_{j=1}^{l}$ and $\gamma$, find

$$
\begin{gathered}
\hat{c}^{*}(\cdot \mid t) \triangleq \arg \min _{J_{i}>0, \hat{c}(\cdot \mid t)} \sum_{j=0}^{N} J_{i} \text { subject to } \\
{\left[\begin{array}{cc}
J_{N} & z^{\prime} \\
z & \gamma^{-1} Q_{j}
\end{array}\right] \geq 0,} \\
\forall z \in \operatorname{vert}\left\{\mathfrak{X}^{t+N \mid t}(x(t))\right\}, \quad j=1, \ldots, l \\
J_{N} \leq \gamma
\end{gathered}
$$

\footnotetext{
${ }^{1}$ This set, according to the $\mathbf{L P V}$ hypothesis reduces to a single point.
}

$$
\begin{gathered}
|F(p(t)) x(t)+\hat{c}(t \mid t)|_{i} \leq u_{i, \max }, \\
\forall i \in\{1, \ldots, m\} \\
\left|F_{j} z(k)+\hat{c}(t+k \mid t)\right|_{i} \leq u_{i, \max }, \\
\forall k \in\{1, \ldots, N-1\}, \\
\forall i \in\{1, \ldots, m\}, \quad \forall j \in\{1, \ldots, l\} \\
\forall z(k) \in \operatorname{vert}\left\{\mathfrak{X}^{t+k \mid t}(x(t))\right\} \\
{\left[\begin{array}{ccc}
1 & x^{\prime}(t) R_{x}^{1 / 2} & \hat{c}^{\prime}(t \mid t) R_{u}^{1 / 2} \\
(*) & J_{0} I & 0 \\
(*) & 0 & J_{0} I
\end{array}\right] \geq 0} \\
\left.\forall \begin{array}{ccc}
1 & z^{\prime}(k) R_{x}^{1 / 2} & \hat{c}^{\prime}(t+k \mid t) R_{u}^{1 / 2} \\
(*) & J_{k} I & 0 \\
(*) & 0 & J_{k} I
\end{array}\right] \geq 0 \\
\forall k \in\{1, \ldots, N-1\}, \forall z(k) \in \operatorname{vert}\left\{\mathfrak{X}^{t+k \mid t}(x(t))\right\}
\end{gathered}
$$

2. Feed the plant by the input

$$
u(t)=F(p(t)) x(t)+c^{*}(t \mid t)
$$

3. $t \leftarrow t+1$, compute $F(p(t)):=\sum_{j=1}^{l} p_{j}(t) F_{j}$ and go to step 1.

Remark 2 - The use of a scheduling control law generates an MPC algorithm having a more demanding computational burden w.r.t. a paradigm which exploits a single linear feedback gain instead. However, the attractiveness characteristics of the proposed algorithm are still intact due to the availability of semidefinite programming solvers [11], capable to treat in an efficient manner optimization problems having a huge number of constraints.

Next lemma ensures that the proposed MPC scheme admits a feasible solution at each time $t$ and the strategy (21) is a stabilizing control law for (1).

Lemma 1 - Let the system (1) be uniformly detectable, then the control strategy (21) is feasible for all $t \in \mathbb{Z}_{+}$, satisfies the prescribed constraints and yields an asymptotically stable closed-loop system provided the Initialization step is solvable.

Proof - Let us suppose $\left\{\left\{P_{j}, F_{j}\right\}_{j=1}^{l}, \gamma\right\}$ be a solution to the Initialization step of DL-LPV and $\left\{c^{*}(t+k \mid t)\right\}_{k=0}^{N-1}$, $\left\{J_{k}^{*}(t)\right\}_{k=0}^{N}$ be the optimal solution of step 1) at the generic time instant $t$. It is straightforward to show (see [3], [4] for details) that the following strategy

$$
\begin{array}{ll}
c(t+1+k \mid t+1) & \leftarrow c^{*}(t+k+1 \mid t), k=0,1, \ldots, N-2, \\
c(t+N-1 \mid t+1) & \leftarrow 0_{m} \\
J_{k}(t+1) \quad \leftarrow \quad & J_{k+1}^{*}(t), \quad k=0,1, \ldots, N-1, \\
J_{N}(t+1) & \leftarrow \quad J_{N}^{*}(t)
\end{array}
$$


is an admissible, tough not optimal, solution for step 1) at the future time instant $t+1$. Moreover, because the following set inclusions are fulfilled along the predictions under (33)-(34)

$$
\mathfrak{X}^{t+k+1 \mid t+1} \subseteq \mathfrak{X}^{t+k+1 \mid t}, \quad k=0,1, \ldots, N-1
$$

the feasibility easily follows. Closed-loop stability of the proposed MPC scheme is proved by showing that the input move

$$
u(t)=F(p(t)) x(t)+c^{*}(t \mid t)
$$

with $c^{*}(t \mid t)$ the first entry of the vector $\left\{c^{*}(t+k \mid t)\right\}_{k=0}^{N-1}$ solution of step 1$)$, satisfies the following property

$$
\lim _{t \rightarrow \infty} c^{*}(t \mid t)=0_{m} .
$$

Because $c^{*}(\cdot \mid t+1)$ needs not to be optimal, it is straightforward to show that

$$
\begin{aligned}
& V(t+1)-V^{*}(t) \leq-\|x(t)\|_{R_{x}}^{2}-\left\|c^{*}(t \mid t)\right\|_{R_{u}}^{2} \\
& +\max _{z \in \operatorname{vert}\left\{\mathfrak{X}^{t+N \mid t}(x(t))\right\}}\|z\|_{R_{x}}^{2} \\
& -\max _{z \in \operatorname{vert}\left\{\mathfrak{X}^{t+N \mid t}(x(t))\right\}, \quad P_{j}, j=1, \ldots, l}\|z\|_{P_{j}}^{2} \\
& +\max _{z \in \operatorname{vert}\left\{\mathfrak{X}^{t+1+N \mid t+1}(x(t+1))\right\}, \quad P_{j}, j=1, \ldots, l}\|z\|_{P_{j}}^{2} .
\end{aligned}
$$

Hence via standard Riccati inequality arguments (see [2]) the sum of the last three terms of the r.h.s. of the inequality (38) is negative definite, and consequently we have

$$
V(t+1)-V^{*}(t) \leq-\|x(t)\|_{R_{x}}^{2}-\left\|c^{*}(t \mid t)\right\|_{R_{u}}^{2} .
$$

This means that the sequence $\left\{V^{*}(t)\right\}_{t=0}^{\infty}$ is monotonically decreasing and admits a unique limit. Therefore

$$
\lim _{t \rightarrow+\infty} c^{*}(t \mid t)=0_{m}
$$

and, as a consequence

$$
\sum_{t=0}^{\infty}\|x(t)\|_{R_{x}}^{2}-\|c(t)\|_{R_{u}}^{2}<\infty .
$$

Hence, $\lim _{t \rightarrow \infty} y(t)=0_{p}, \lim _{t \rightarrow \infty} c(t)=0_{m}$ and, by detectability condition, $\lim _{t \rightarrow \infty} x(t)=0_{n}$.

Remark 3 - Note that the proposed MPC strategy DL-LPV is also applicable, mutatis mutandis, if the LPV hypothesis is removed (see [6] and [13]). In this case, the parameter $p(t)$ is not directly available and the proposed control law has no longer a scheduling structure and reduces to

$\hat{u}(\cdot \mid t)= \begin{cases}F \hat{x}(t+k \mid t)+\hat{c}(t+k \mid t), & k=0,1, \ldots, N-1 \\ F \hat{x}(t+k \mid t), & k \geq N\end{cases}$

where $F=Y G^{-1}$. Therefore, the algorithm becomes as follows:

\section{Algorithm - DL-Robust}

- Initialization:

Given $x(0)$ find

$$
\left[\left\{Q_{j}\right\}_{j=1}^{l}, Y, G, \gamma\right]:=\arg \min _{Y, Q_{j}>0, \gamma>0} \gamma
$$

subject to LMIs (17)-(19) with the constraints $Y_{i}=$ $Y, i=1, \ldots, l, j=i$.

$P_{j}:=\gamma Q_{j}^{-1}, j=1, \ldots, l, F:=Y G^{-1}$.

- Generic Step:

1. For any $t \geq 0$, given $x(t),\left\{P_{j}\right\}_{j=1}^{l}, F$, and $\gamma$, find

$$
\hat{c}^{*}(\cdot \mid t) \triangleq \arg \min _{J_{i}>0, \hat{c}(\cdot \mid t)} \sum_{j=0}^{N} J_{i} \text { subject to }
$$

$$
\begin{aligned}
& {\left[\begin{array}{cc}
J_{N} & z^{\prime} \\
z & \gamma^{-1} Q_{j}
\end{array}\right] \geq 0,} \\
& \forall z \in \operatorname{vert}\left\{\mathfrak{X}^{t+N \mid t}(x(t))\right\}, \quad j=1, \ldots, l \\
& J_{N} \leq \gamma \\
& |F z(k)+\hat{c}(t+k \mid t)|_{i} \leq u_{i, \max }, \\
& \forall k \in\{0,1, \ldots, N-1\}, \\
& \forall i \in\{1, \ldots, m\} \\
& \forall z(k) \in \operatorname{vert}\left\{\mathfrak{X}^{t+k \mid t}(x(t))\right\} \\
& {\left[\begin{array}{ccc}
1 & z^{\prime}(k) R_{x}^{1 / 2} & \hat{c}^{\prime}(t+k \mid t) R_{u}^{1 / 2} \\
(*) & J_{k} I & 0 \\
(*) & 0 & J_{k} I
\end{array}\right] \geq 0} \\
& \forall k \in\{0,1, \ldots, N-1\}, \\
& \forall z(k) \in \operatorname{vert}\left\{\mathfrak{X}^{t+k \mid t}(x(t))\right\}
\end{aligned}
$$

2. Feed the plant by the input

$$
u(t)=F x(t)+c^{*}(t \mid t)
$$

3. $t \leftarrow t+1$ and go to step 1 .

Note finally that, due to the removal of the LPV hypothesis, the structure of the sets $\mathfrak{X}^{t+k \mid t}(x(t)), k=1, \ldots, N$, is drastically simplified because the closed loop matrix $A_{F}(t)$ belongs to

$$
A_{F}(t) \in \operatorname{co}\left\{\bar{A}_{i}, \quad i=1, \ldots, l\right\}
$$

where

$$
\bar{A}_{i}:=A_{i}+B_{i} F
$$

\section{NUMERICAL EXPERIMENTS}

The aim of this section is to test the effectiveness of the proposed on-line MPC strategy. To this end, the scheme will be compared in terms of both control performances and computational complexity with the algorithm given in [4] (NDL-LPV), based on standard quadratic stabilizability conditions and with the robust dilated-based on-line MPC scheme of [6] (algorithm DL-Robust, see Remark 1). All the computations, have been carried out on a Pentium 4 using the YALMIP Toolbox [11]. 


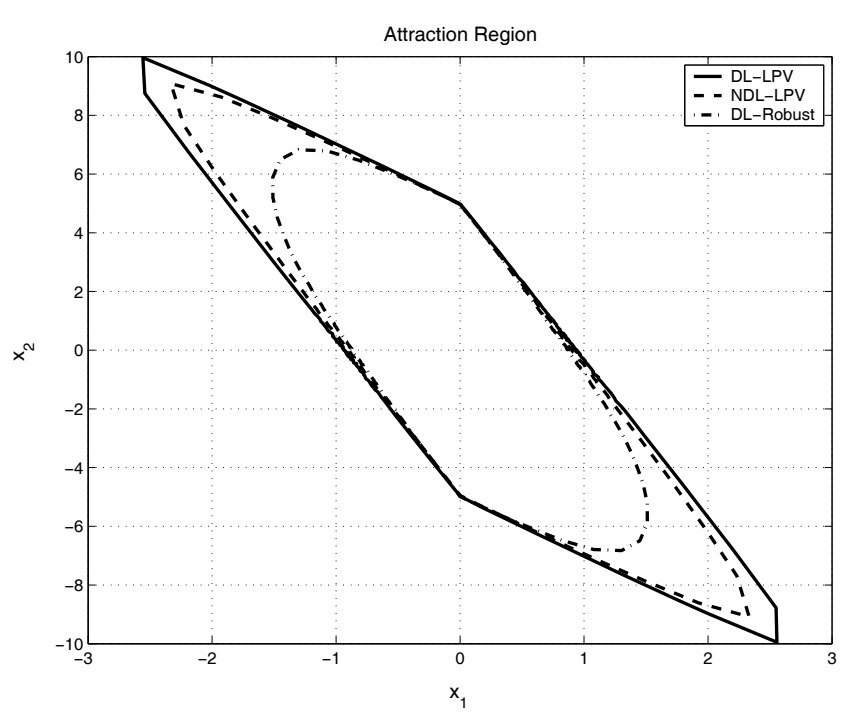

Fig. 1. Attraction Regions - DL-LPV (Continuous line), NDL-LPV (Dashed line), DL-Robust (Dash-Dotted line)

\section{A. Experiment 1}

Consider the polytopic uncertain system

$$
\begin{aligned}
x(t+1) & =\sum_{i=1}^{2} p_{i}(t) A_{i} x(t)+\sum_{i=1}^{2} p_{i}(t) B_{i} u(t) \\
y(t) & =C x(t)
\end{aligned}
$$

where the system matrix vertices are

$$
\begin{aligned}
& A_{1}=\left[\begin{array}{ll}
1 & 0.1 \\
0.5 & 1
\end{array}\right] \\
& A_{2}=\left[\begin{array}{ll}
1 & 0.1 \\
2.5 & 1
\end{array}\right] \\
& B_{1}=B_{2}=\left[\begin{array}{l}
1 \\
0
\end{array}\right] .
\end{aligned}
$$

and the input $u(t)$ is subject to the following saturation constraints

$$
|u(t)| \leq 0.5, \quad \forall t \geq 0 .
$$

The parameter vector $p(t)$ is assumed to be measurable at each time instant $t$ and, for simulation purposes, we have assumed that $p(t)=\left[\begin{array}{ll}0.3,0.7\end{array}\right], \forall t$. The MPC algorithm DL-LPV has been solved for $N=2$. In order to observe the benefits, in the following Figures 1, 2 the attraction region and the regulated state response along with the control input are depicted for 10 sampling steps: the dashed and dashdotted graphs represent the control actions of NDL-LPV and, respectively, DL-Robust whereas the continuous line represents the action of DL-LPV.

Finally, in Figure 3, the guaranteed upper-bound to the quadratic cost for the three schemes is reported.

It can be observed in Figure 1, that, thanks to less conservative stability conditions, DL-LPV exhibits a larger attraction basin. For the same reason, in Figure 2, the
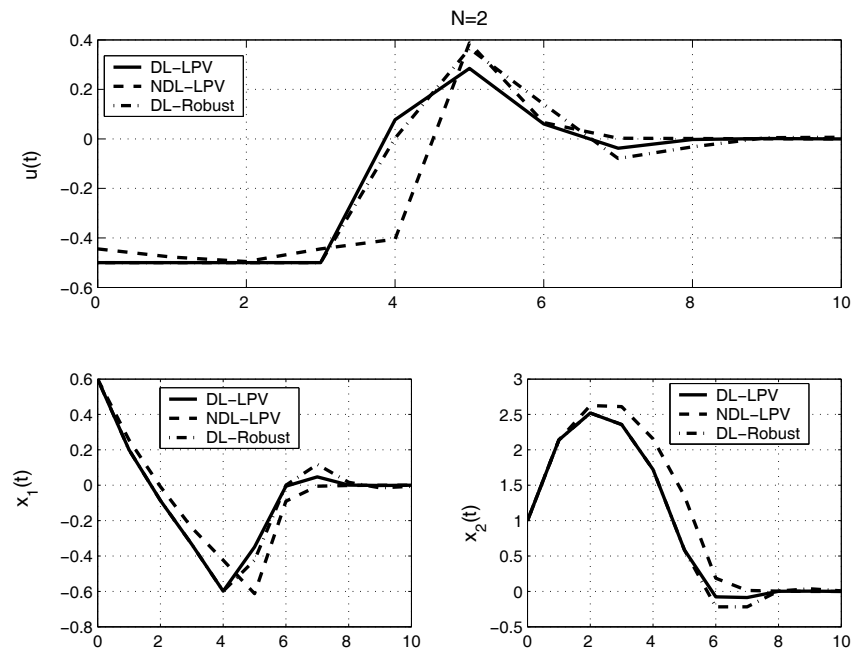

Fig. 2. Regulated state trajectories and control input, $N=2, x(0)=$ $\left[\begin{array}{ll}0.6 & 1\end{array}\right]^{T}$, - DL-LPV (Continuous line), NDL-LPV (Dashed line), DLRobust (Dash-Dotted line)

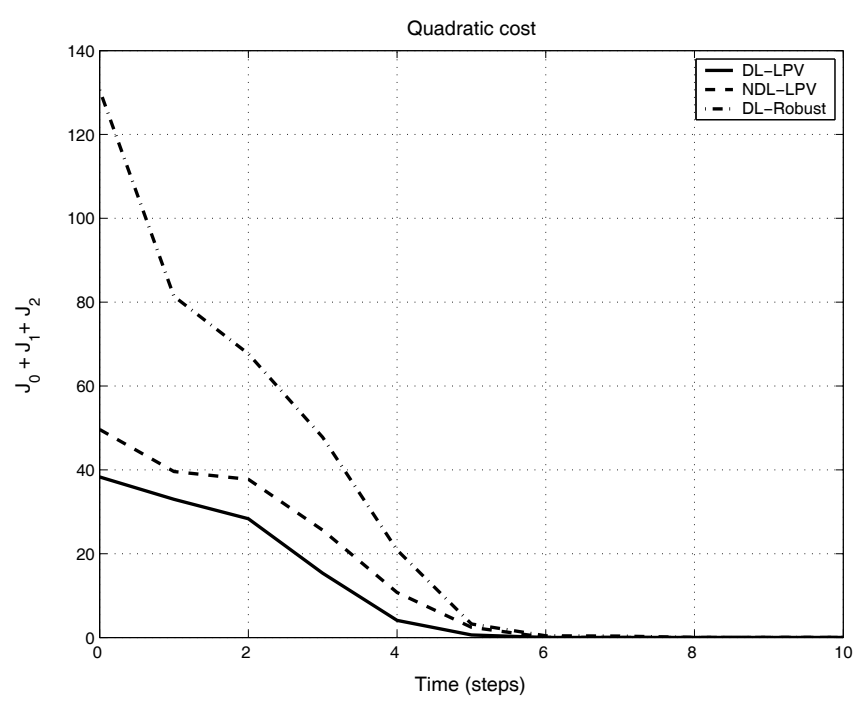

Fig. 3. Upper bound to the guaranteed cost, $N=2$, - DL-LPV (Continuous line), NDL-LPV (Dashed line), DL-Robust (Dash-Dotted line)

dilated schemes DL-LPV and DL-Robust, exhibit better regulated responses w.r.t. NDL-LPV, especially in terms of control action for the first time instants. A look at Figure 3 reveals instead that the proposed control strategy DL-LPV achieves the prescribed regulation results at the expense of a significantly lower guaranteed cost.

The computational effort for the three algorithms is reported in the following Table I. As expected, due to the presence of a higher number of constraints and due to the scheduling structure, the proposed DL-LPV algorithm shows an on-line computational burden that is only slightly worse than the others. 
TABLE I

ON-LINE NUMERICAL BURDENS: AVERAGE CPU TIME (SECS.) PER STEP

\begin{tabular}{|c||c|}
\hline Algorithm & CPU time (average) \\
\hline DL-LPV & 0.475 \\
\hline NDL-LPV & 0.354 \\
\hline DL-Robust & 0.446 \\
\hline
\end{tabular}

\section{B. Experiment 2}

The following example is taken from [3] where a robust servomechanism is designed for a polytopic uncertain system subject to a previewable reference signal. In particular, the plant output is required to track an assigned reference trajectory $y_{r}(t)=C_{r} x_{r}(t)$, whose samples are computed from the state of the autonomous system (signal generator)

$$
x_{r}(t+1)=A_{r} x_{r}(t), \quad x_{r}(0)=x_{r 0}
$$

and a certain number of future samples of the reference signal are available at each time instant for predictions.

The overall control procedure, presented in the previous sections for regulation problems, can also be used to solve tracking problems by resorting to incremental system description and by using a suitably augmented state (see [1] and [19] for details). We consider the following discrete-time LPV system

$$
\left\{\begin{array}{l}
A(p)=\left[\begin{array}{cc}
0.5 & 1 \\
0.25 & 1.5
\end{array}\right] p_{1}+\left[\begin{array}{cc}
1.5 & 1 \\
0.75 & 0.2
\end{array}\right] p_{2} \\
B(p)=\left[\begin{array}{l}
0 \\
1
\end{array}\right] p_{1}+\left[\begin{array}{l}
1 \\
1
\end{array}\right] p_{2} \\
C=\left[\begin{array}{ll}
0 & 1
\end{array}\right]
\end{array}\right.
$$

with $p=\left[p_{1}, p_{2}\right]$ in the simplex unit of $\mathbb{R}^{2}$ having the following structure

$$
p(t):=\left\{\begin{array}{l}
p_{1}(t)=p_{2}(t)=0.5 \\
0 \leq t<30 \mathrm{sec} \\
p_{1}(t)=0.5+0.01 t, \quad p_{2}(t)=0.5-0.01 t \\
30 \leq t<45 \mathrm{sec} \\
\\
p_{1}(t)=0.8-0.01 t, \quad p_{2}(t)=0.2+0.01 t \\
t \geq 45 \mathrm{sec}
\end{array}\right.
$$

An incremental augmented model with preview length $h=2$ has been built up. The state and input weighting matrices has been chosen equal to $R_{x}=1, R_{u}=\operatorname{diag}\left(1,0_{2 \times 2}, 0_{2 \times 2}\right)$. The reference signal is the following sinusoidal wave $r(t)=$ $\sin \left(\frac{\pi}{30} t\right)$, and the constraint on the control input increments has been chosen equal to $|u(t+1)-u(t)| \leq 0.3$.

In Figure 4, for the proposed MPC scheme DL-LPV, the tracking performances in terms of regulated trajectory and incremental input for control horizons $N=2,4$ are depicted for a simulation interval of 60 time units. It appears that increasing $N$ implies better tracking performances in terms of tracking error and reduction of phase lag.
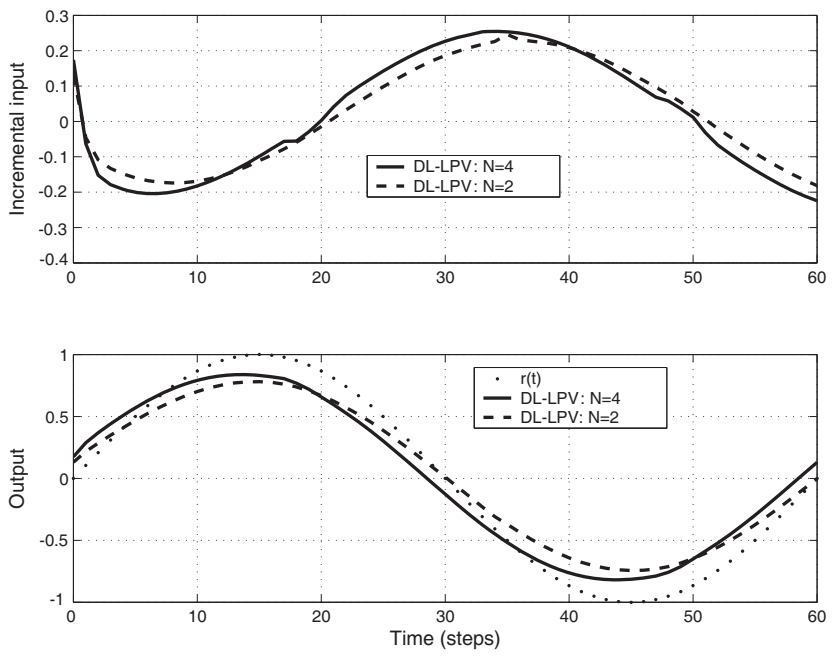

Fig. 4. Tracking Output and Incremental Control, Algorithm DL-LPV $N=2$ (Dashed line graph), $N=4$ (Continuous line graph)
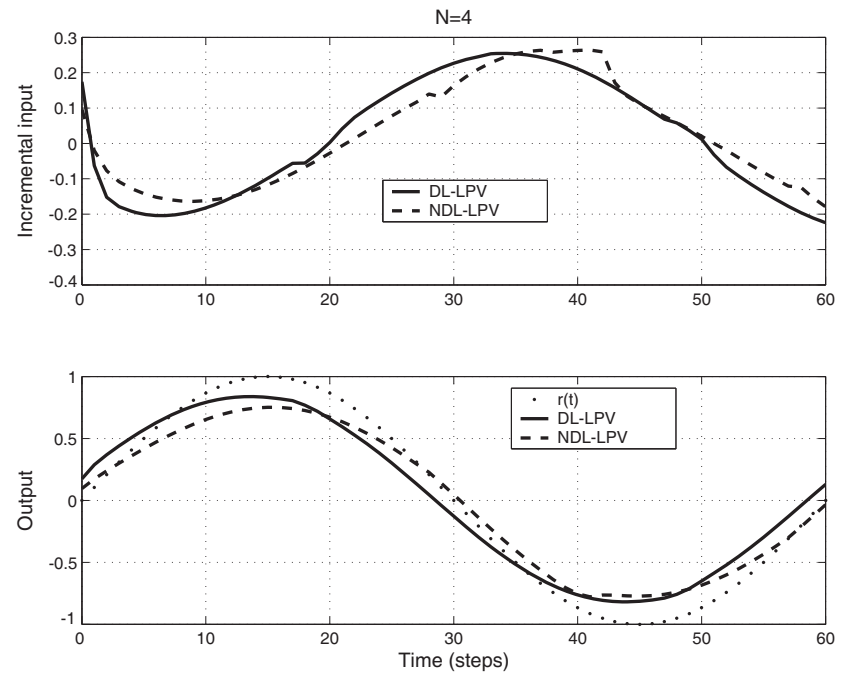

Fig. 5. Tracking Output and Incremental Control, Comparison between DLLPV (Continuous line graph) and NDL-LPV (Dashed line graph), $N=4$

In Figure 5, the DL-LPV MPC strategy is compared in terms of regulated responses w.r.t. its NDL-LPV counterpart for a control horizon $N=4$. The simulation parameters are the same of the previous experiments. It can be noted that the proposed dilated scheme exhibits better tracking response and an incremental input whose characteristics are similar w.r.t. to the one computed by the NDL-LPV algorithm, except for the first 10 time units, where the dilated control signal is more active.

\section{CONCLUSion}

In this paper, a dilation-based LPV predictive control strategy has been presented for discrete-time systems described by polytopic (multi-model) representations. The LPV parameter has been assumed measurable and we have considered a stabilizing scheduling state-feedback controller. The 
main contribution of the proposed strategy is to extend the RHC scheme presented in [5] to the general case of control horizons of arbitrary length $N$.

The improvement accomplished with this MPC scheme w.r.t. standard LPV algorithms, which make use of quadratic stability arguments [4], relies on the use of parameterdependent Lyapunov functions which enable to redefine less conservatively the set of all $k$-steps ahead state predictions.

Numerical experiences have shown performance improvements in terms of control input activity and quadratic cost reduction when the algorithm is compared to the non-dilated LPV scheme. A similar behavior has been also observed when numerical comparisons have been performed w.r.t. an on-line robust dilated paradigm by discarding the LPV hypothesis.

\section{REFERENCES}

[1] D. Angeli, A. Casavola and E. Mosca, Predictive PI-control under positional and incremental input saturations, Automatica, Vol. 36, 2000, pp. 151-156.

[2] S. Boyd, L. El Ghaoui, E. Feron and V. Balakrishnan, Linear Matrix Inequalities in System and Control Theory, SIAM Studies in Applied Mathematics, 15, SIAM, London.

[3] A. Casavola, D. Famularo and G. Franzè, A Feedback MinMax MPC Algorithm for LPV Systems subject to Bounded Rates of Change of Parameters, IEEE Transaction on Automatic Control, Vol. 47, No. 7, 2002, pp. 1147-1153.

[4] A. Casavola, D. Famularo and G. Franzè, Predictive control of constrained nonlinear system via LPV linear embeddings, Int. J. Robust Nonlinear Control, Vol. 13, 2003, pp 281-294.

[5] A. Casavola, D. Famularo, G. Franzè and E. Garone, An improved predictive control strategy for polytopic LPV linear systems, Proceedings of the 45th IEEE Conference on Decision and Control, San Diego, CA, 2006.

[6] F.A. Cuzzola, J.C. Jeromel and M. Morari, An improved approach for contrained robust model predictive control, Automatica, Vol. 38, 2002, pp. 1183-1189.
[7] J. Daafouz and J. Bernussou, Parameter dependent Lyapunov functions for discrete time systems with time varying parametric uncertainties,System \& Control Letters, Vol. 43, 2001, pp. 355-359.

[8] M.C. de Oliveira, J. Bernussou, and J.C. Geromel, A new discrete-time robust stability condition,System \& Control Letters, Vol. 37, 1999, pp. 261-265.

[9] J.C. Geromel, P.L.D. Peres and J. Bernussou, On a convex parameter space method for linear control design of uncertain sstems, SIAM Journal of Control and Optimization, Vol. 29, 1991, pp. 381-402.

[10] M.V. Kothare, V. Balakrishnan and M. Morari, Robust constrained model predictive control using linear matrix inequalities, Automatica, Vol. 32, 1996, pp. 1361-1379.

[11] J. Löfberg. A Toolbox for Modeling and Optimization in MATLAB. Proceedings of the (CACSD) Conference. Taipei, Taiwan, 2004. Available from http://control.ee.ethz.ch/ joloef/yalmip. php.

[12] Y. Lu and Y. Arkun, Quasi-min-max MPC algorithms for LPV systems, Automatica, Vol. 36, pp. 527-540, 2000.

[13] Wei-Jie Mao, Robust stabilization of uncertain time-varying discrete systems and comments on "an improved approach for constrained robust model predictive control", Automatica, Vol. 39, 2003, pp. 11091112.

[14] D.Q. Mayne, J.B. Rawlings, C.V. Rao and P.O.M. Scokaert, Constrained model predictive control: stability and optimality, Automatica, Vol. 36, 2000, 789-814.

[15] D.Q. Mayne, M.M. Seron and S.V. Rakovic, Extended invariance and its use in model predictive control, Automatica, Vol. 41, 2005, 21632169.

[16] P. Park and S. C. Jeong, Constrained RHC for LPV systems with bounded rates of parameter variations, Automatica, Vol. 40, 2004, pp. 865-872.

[17] J. B. Rawlings, K. R. Muske, The stability of constrained receding horizon control, IEEE Transactions on Automatic Control, Vol. 38, 1993, pp. 1512-1516.

[18] J. A. Rossiter, B. Kouvaritakis and M. J. Rice, A numerically robust state-space approach to stable-predictive control strategies, Automatica, Vol. 34, 1998, pp. 65-74.

[19] K. Takaba. "Robust servomechanism with preview action for polytopic uncertain systems". International Journal of Robust and Nonlinear Control, Vol. 10, pp. 101-111,2000. 